Int. J. Odontostomat., 9(1):31-35, 2015.

\title{
Sarcoma Pleomórfico Indiferenciado de Maxilar de Alto Grado: Diagnóstico, Tratamiento y Pronóstico
}

\author{
Undifferentiated Pleomorphic Sarcoma of the Maxillary: \\ Diagnosis, Treatment and Prognosis
}

Daniel Betancur Castro*; Milly Yáñez Morales** \& Sergio Moya Pradena**

BETANCUR, C. D.; YÁÑEZ, M. M. \& MOYA, P. S. Sarcoma pleomórfico indiferenciado de maxilar de alto grado. Diagnóstico, tratamiento y pronóstico. Int. J. Odontostomat., 9(1):31-35, 2015.

RESUMEN: Los sarcomas son neoplasias poco vistas en el territorio maxilofacial, donde la baja frecuencia de casos y los cambios de nomenclatura, han dificultado su estudio. Una de las variables de este tipo de lesiones corresponde a un tumor conocido como sarcoma pleomórfico indiferenciado, del cual se sabe muy poco pues se describe una incidencia de 1:1.000.000. Corresponde a un tumor maligno con características clínicas e imagenológicas de crecimiento invasivo y destructivo; la confirmación diagnóstica se realiza por medio de una biopsia en base al estudio histológico e inmunohistoquímico. Aparece principalmente en el tracto nasosinusal y su manejo terapéutico se basa en la cirugía con amplios márgenes de seguridad y radioterapia o quimioterapia según corresponda. No presenta metástasis locales pero si a distancia con gran afinidad por el pulmón. Se reporta un caso de sarcoma pleomórfico indiferenciado de alto grado de maxilar diagnosticado en la Unidad de Patología Oral y Maxilofacial del Hospital las Higueras de Talcahuano durante el año 2014, que a diferencia de la mayoría de los casos reportados, producto de su extensión, fue tratado con radioterapia paliativa. Además, se realizó una revisión de la literatura disponible en diversas bases de datos con lo cual se obtuvo información de casos ya reportados. Actualmente resulta difícil producto de la baja cantidad de casos descritos poder hacer proyecciones en cuanto a su pronóstico o evolución, sin embargo hay consenso de que el manejo interdisciplinario, diagnóstico precoz y la confirmación de este por medio de exámenes complementarios son fundamentales para su adecuado tratamiento.

PALABRAS CLAVE: sarcoma, cabeza y cuello, sarcoma pleomorfo indiferenciado.

\section{INTRODUCCIÓN}

Los sarcomas corresponden a una patología neoplásica infrecuente, que puede adoptar variados patrones morfológicos en base a la estirpe celular del cual proceden y el grado de diferenciación que alcanzan. En el área de cabeza y cuello estos tumores muy poco comunes, alcanzan solo el $1 \%$ de los sarcomas primarios (Shellenberger \& Sturgis, 2009), y entre el 4 y $10 \%$ de los sarcomas en general, todo ellos con origen en células mesenquimáticas (Ketabchi et al., 2011).

Este tipo de tumores resultan complejos en el área maxilofacial, puesto que si bien han sido estudiados en mayor profundidad en otras regiones como miembros y tronco, los conceptos generales sobre el manejo de sarcomas no son universalmente aplicados en cabeza y el cuello (Barosa et al., 2014). Estas neoplasias puede tender a la malignización, en la medida que pierden las características de diferenciación celular propias de su célula de origen, con presencia de pleomorfismos y altos grados de indiferenciación, un ejemplo de aquello es un tumor conocido como sarcoma pleomórfico indiferenciado.

El sarcoma pleomorfo indiferenciado, antes nombrado como histiocitoma fibroso maligno, se ha establecido como una entidad patológica desde el año 2002 en la clasificación de la OMS de tumores de par-

\footnotetext{
Facultad de Odontología Universidad de Concepción, Concepción, Chile.

* Departamento de Anatomía Patológica y Patología Oral y Maxilofacial, Hospital las Higueras de Talcahuano, Talcahuano, Chile.

**** Unidad de Cirugía Maxilofacial Hospital las Higueras de Talcahuano, Talcahuano, Chile.
} 
tes blandas. Antiguamente la descripción de "histiocitoma fibroso maligno" abarcaba la mayoría de los tumores malignos de tejidos blandos que no tenían componentes distintivos, incluyéndose así aproximadamente al $40 \%$ de estos; sin embargo, con el cambio de nomenclatura propuesto por la OMS solo alcanzan el $5 \%$, y los tumores que surgen en la cabeza y el cuello contribuyen sólo alrededor de 1-3\% de todos los sarcomas pleomórficos indiferenciados (MüllerRichter et al., 2008).

La etiología exacta de los sarcomas de cabeza y cuello se desconoce, sin embargo existe una tendencia a que podría ser idiopática, haber una predisposición genética, exposición a radiación, agentes virales y químicos cancerígenos (Aljabab et al., 2011).

El diagnóstico se basa en las características e historia clínica de la lesión, apoyada en estudios imaginológicos, histológicos e inmunohistoquímicos. Clínicamente corresponden a un aumento de volumen cubierto por mucosa, de crecimiento rápido e infiltrativo, que no causan mayor dolor. Se presenta como una masa sésil, suave y elástica con características osteolíticas y bordes bien definidos, pudiendo ser intra o extraóseos (Tanaka et al., 2011). Histológicamente hay células fusadas, pleomórficas y mitosis atípicas, al estudio inmunohistoquímico resulta positivo para vimentina, un marcador con gran afinidad para sarcomas y tejidos mesenquimáticos (Müller-Richter et al.; Tanaka et al.).

Los estudios imagenológicos indicados son la resonancia nuclear magnética y la tomografía computadorizada (TC), donde se observa como una masa sólida, con un centro atenuado debida a la necrosis, pudiendo haber hemorragia o presencia de material mixoide (Park et al., 2009). En la resonancia nuclear magnética se ve una intensidad de señal heterogénea, lo que refleja los componentes histológicos complejos del tumor, sin haber diferencias significativas entre los subtipos de este. Se aprecia como origen más frecuente el tracto nasosinusal, y tanto en TC como en resonancia nuclear magnética se evidencia invasión de tejidos blandos y destrucción ósea, con aspecto similar a músculo (Vuity et al., 2013).

El diagnóstico definitivo se basa en el examen histopatológico, por medio de una biopsia incisional, aun cuando el alto grado de vascularización de este tipo de lesiones puede dificultar su obtención.

El camino para llegar a un sarcoma pleomórfico indiferenciado, considera una pérdida de la diferencia- ción de las células que originalmente formaron el tumor, llegando a un punto en que no puede ser reconocida la estirpe celular inicial, lo que se evidencia al dar positivo para marcadores de p53, que es un marcador tumoral, y Ki-67, que es indicativo de proliferación celular; algunos investigadores como Tanaka et al. han informado que esta malignización podría ser secundaria a un trauma o una infección crónica, donde la hipótesis se sustenta en la llegada de fibrocitos y otras células madres (indiferenciadas) al sitio de la lesión. A nivel molecular, se cree habría alteraciones a nivel de los genes INK4A y INK4B, de la ciclina D1 y del cromosoma 17 (Aljabab et al.).

El manejo terapéutico de estos cuadros se resume principalmente a la resección quirúrgica complementada con radioterapia pre o postoperatoria o quimioterapia según las características del tumor.

La mayoría de los sarcomas pleomórficos indiferenciados recidivan localmente, siendo las metástasis a distancia más comunes que las locales y con mayor frecuencia en pulmón (Vuity et al.).

\section{PRESENTACIÓN DEL CASO}

Paciente sexo masculino de 69 años, sin antecedentes médicos previos, consulta por aumento de volumen, de 5 meses de evolución en zona posterior del maxilar izquierdo luego de una exodoncia del diente 27 , la cual presentaba notable movilidad y había sido diagnosticada con periodontitis crónica marginal (Fig. 1).

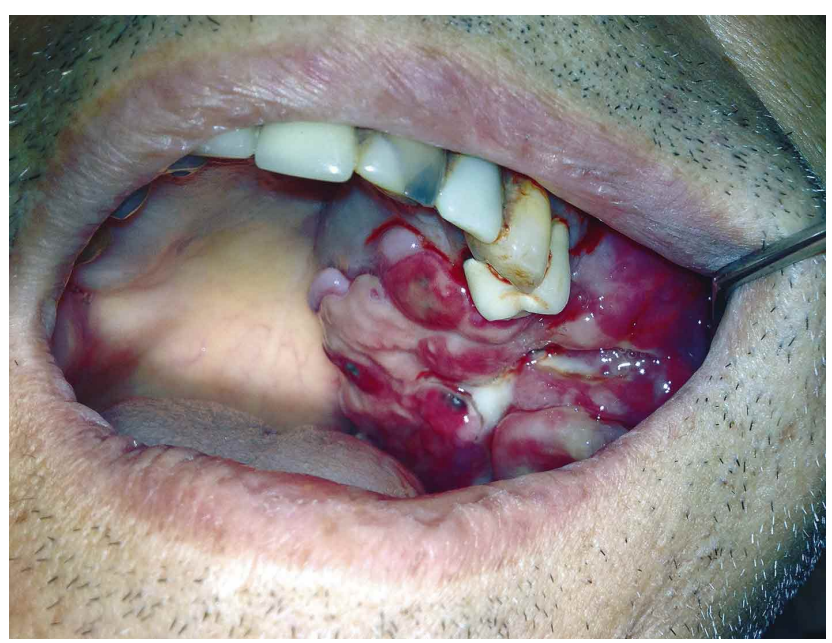

Fig. 1. Presentación clínica del aumento de volumen, de 5 meses de evolución en zona posterior del maxilar izquierdo luego de una exodoncia del diente 27. 


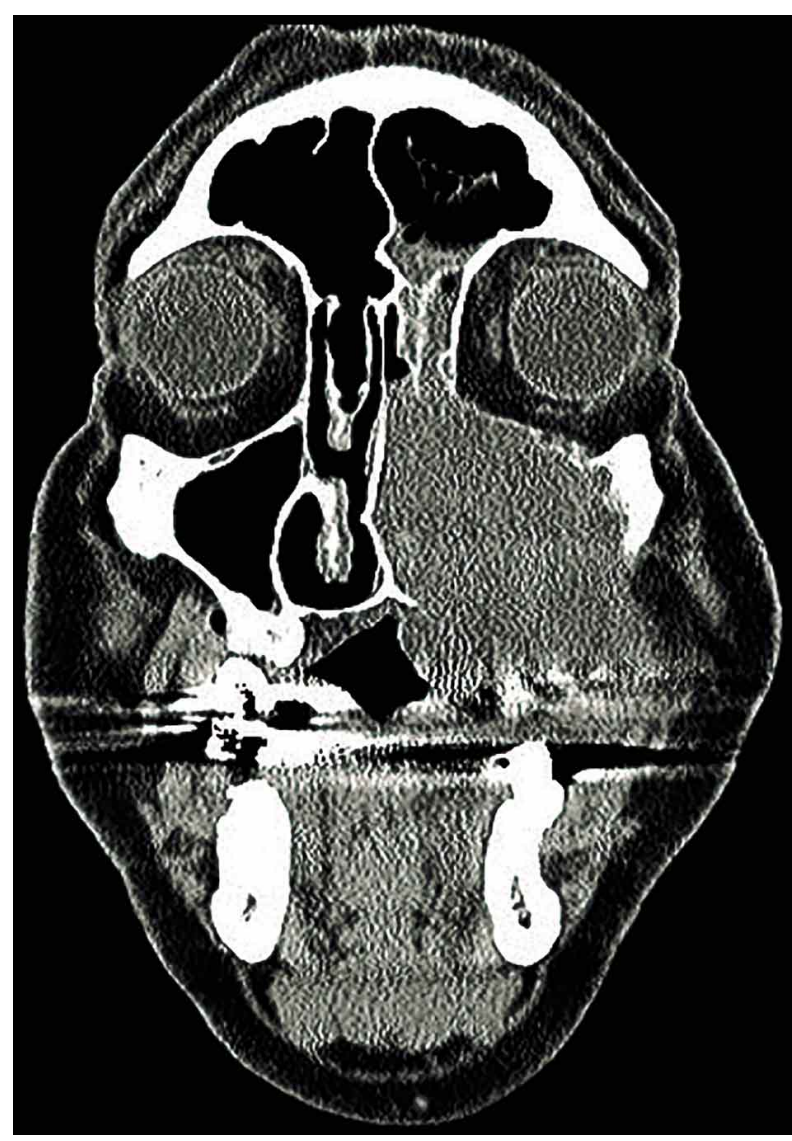

Fig. 2. Corte coronal de Tomografía computadorizada donde se observa una masa sólida expansiva ocupando el seno maxilar izquierdo, comprometiendo piso de orbita y seno esfenoidal.

Al examen clínico, se observaba una masa sésil, cubierta por mucosa con zonas ulceradas, indolora, con leve hemorragia y de consistencia elástica. Se indica una radiografía panorámica la cual, tras presentar una zona radiopaca difusa en relación a la zona del diente 27 fue complementada con una TC.

En la TC se evidencia una masa sólida expansiva ocupando todo el seno maxilar izquierdo, comprometiendo piso de orbita y seno esfenoidal, que capta en forma heterogénea contraste e infiltra planos grasos y musculares vecinos con destrucción del hueso adyacente. Presenta $7,7 \mathrm{~cm}$ en el eje mayor y no se visualizan linfonodopatías (Fig. 2).

Se realizó biopsia para estudio histológico, donde se observo con tinción de hematoxilina eosina, presencia de pleomorfismo celular, con células sin características de diferenciación evidentes, de aspecto fusiforme en una matriz aparentemente mixoide. La confirmación diagnóstica se realizó por medio de una batería de marcadores inmunohistoquímicos dando positivo para vimentina y negativo para actina, CD34, CD68, CK7, CK20, desmina, HMB45, KE13, melanina y $\$ 100$, salvo Ki67 para el cual dio positivo en un más de un 8\% (Fig. 3 ), por lo que se diagnosticó como sarcoma pleomórfico indiferenciado de alto grado, de maxilar; además se realizó radiografía de tórax con lo cual se descartó la presencia de metástasis pulmonares a distancia.

Producto del tamaño y ubicación de la lesión se descartó el tratamiento quirúrgico en una primera instancia y se planificó radioterapia paliativa, con una dosis de entrada de 50 a 60 Grey. Luego de 40 sesiones de radioterapia, el tumor remite considerablemente; se indicó control al mes para análisis de un esquema quimioterapéutico paliativo, sin embargo, aun cuando el tumor remitía de forma favorable, el paciente fallece a causa de una neumonía aguda y deterioro pulmonar pocos días después de finalizada la radioterapia.

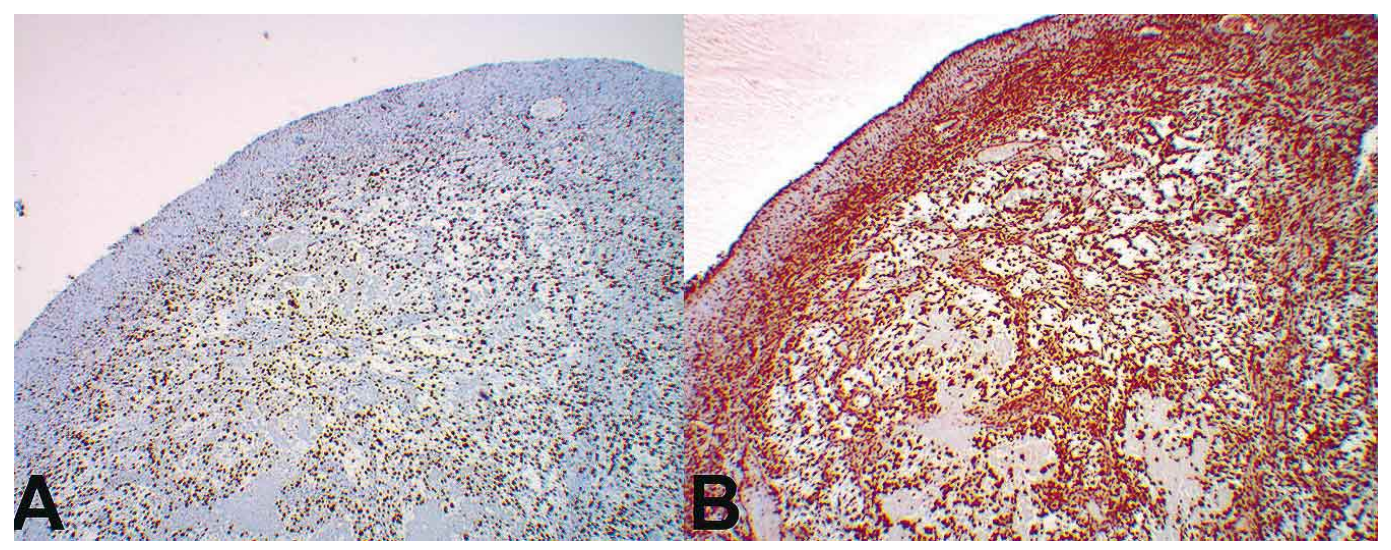

Fig. 3. Cortes histológicos de la biopsia. A, tinción inmunohistoquímica positiva para Ki67. B, tinción de hematoxilina eosina donde se observa presencia de pleomorfismo celular, con células sin características de diferenciación evidentes, de aspecto fusiforme en una matriz aparentemente mixoide. 


\section{DISCUSIÓN}

El manejo terapéutico de estos cuadros se resume principalmente a la resección quirúrgica complementada con radioterapia o quimioterapia según las características del tumor.

La resección quirúrgica es difícil de conseguir, aumentando el riesgo de recidivas, además la delicada anatomía de la cabeza y el cuello limita la capacidad de obtener márgenes quirúrgicos amplios, razón por la cual hay una mayor tasa de recidiva local en sarcomas de cabeza y cuello en comparación con otros sitios (de Bree et al., 2010; Modesto et al., 2014; Mattavelli et al., 2013). La mayoría de los sarcomas pleomórficos indiferenciados recidiva localmente, siendo las metástasis a distancia más comunes que las locales y con mayor frecuencia en pulmón (Vuity et al.).

Respecto a la etapa en la cual aplicar la radioterapia, la incidencia de complicaciones microvasculares y de la cicatrización no fue significativamente diferente pre y post quirúrgicas, sin embargo, existen controversia en estudios que demuestran que los pacientes que recibieron radioterapia preoperatoria experimentaron menos complicaciones del sitio que aquellos que recibieron terapia de radiación postoperatoria (Chao et al., 2013).
Producto del alto grado de vascularización de este tipo de lesiones, es importante considerar algunos aspectos en su manejo como la posibilidad de utilizar biopsia por aspiración, la cual es una técnica poco invasiva con una sensibilidad de un 60 a $80 \%$ (Sarrami et al.), que reduciría el riesgo de complicaciones hemorrágicas durante la obtención de la muestra.

Se sugiere además revisar la literatura respecto a que en el caso de los tumores de cabeza y cuello se pueda considerar, a la hora de descartar la existencia de metástasis pulmonares, extender la TC con contraste de cabeza y cuello hasta la región torácica, con el fin de poder en un mismo examen de alta precisión descartar con mayor seguridad la presencia de metástasis a distancia.

En cuanto al pronóstico de estas lesiones, debido a la rareza en los adultos y el pequeño número de centros de tratamiento que reportan dichos casos, no hay datos clínicos basados en la evidencia suficiente para proporcionar un número de pacientes que permita identificar factores pronósticos o influencias asociadas al grado de supervivencia (Mucke et al., 2010). No obstante hay consenso en que su manejo debe ser multidisciplinario (Tejani et al., 2013).

BETANCUR, C. D.; YÁÑEZ, M. M. \& MOYA, P. S. Undifferentiated pleomorphic sarcoma, of the maxillary: diagnosis, treatment and prognosis. Int. J. Odontostomat., 9(1):31-35, 2015.

ABSTRACT: Sarcomas are neoplastic lesions rarely observed in the maxillofacial region. Due to the few cases reported and to changes in nomenclature of these tumors their study has been difficult. One of the variants of this kind of lesion corresponds to a tumor currently known as undifferentiated pleomorphic sarcoma, which there is scarce knowledge of. In fact the incidence described is about 1:1.000.000. This one corresponds to a malignant tumor with image and clinical features as invasive and destructive growth, the confirmation of diagnosis is carried out by performing biopsy based on histologic and immunohistochemical studies. They appear mostly in the nose and sinusal tract. Its therapeutic management is based on surgery with wide security margins and radio or chemotherapy when warranted. No local metastases but distant ones are found and highly affined with lungs. We report a case of an advanced undifferentiated pleomorphic sarcoma diagnosed at the Oral and maxillofacial pathology unit of Hospital Las Higueras in Talcahuano at 2014, which in spite of most of the reported cases in literature; because of the extent it was treated with palliative radiotherapy. Also has been made review of the available literature in different databases with which information of the reported cases was obtained. Nowadays, due to the low number of cases described it is difficult to establish projections in terms of prognosis or evolution. However, there is consensus that interdisciplinary management, early diagnosis and confirmation with complementary exams are essential for an appropriate treatment.

KEY WORDS: sarcoma, head and neck, undifferentiated pleomorphic sarcoma. 


\section{REFERENCIAS BIBLIOGRÁFICAS}

Aljabab, A. S.; Nason, R. W.; Kazi, R. \& Pathak, K. A. Head and neck soft tissue sarcoma. Indian J. Surg. Oncol., 2(4):286-90, 2011.

Barosa, J.; Ribeiro, J.; Afonso, L.; Fernandes, J. \& Monteiro, E. Head and neck sarcoma: analysis of 29 cases. Eur. Ann. Otorhinolaryngol. Head Neck Dis., 131(2):83-6, 2014.

Chao, A. H.; Sturgis, E. M.; Yu, P.; Skoracki, R. J.; Guadagnolo, B. A. \& Hanasono, M. M. Reconstructive outcomes in patients with head and neck sarcoma. Head Neck, 35(5):677-83, 2013.

de Bree, R.; van der Waal, I.; de Bree, E. \& Leemans, C. R. Management of adult soft tissue sarcomas of the head and neck. Oral Oncol., 46(11):786-90, 2010.

Ketabchi, A.; Kalavrezos, N. \& Newman, L. Sarcomas of the head and neck: a 10-year retrospective of 25 patients to evaluate treatment modalities, function and survival. Br. J. Oral Maxillofac. Surg., 49(2):116-20, 2011.

Mattavelli, D.; Miceli, R.; Radaelli, S.; Mattavelli, F.; Cantù, G.; Barisella, M.; Quattrone, P.; Stacchiotti, S.; Sangalli, C.; Casali, P. G.; Gronchi, A. \& Fiore, M. Head and neck soft tissue sarcomas: prognostic factors and outcome in a series of patients treated at a single institution. Ann. Oncol., 24(8):2181-9, 2013.

Modesto, A.; Filleron, T.; Chevreau, C.; Le Pechoux, C.; Rochaix, P.; Le Guellec, S.; Ducassou, A.; Gangloff, D.; Ferron, G. \& Delannes, M. Role of radiation therapy in the conservative management of sarcoma within an irradiated field. Eur. J. Surg. Oncol., 40(2):187-92, 2014.

Mucke, T.; Mitchell, D. A.; Tannapfel, A.; Hölzle, F.; Kesting, M. R.; Wolff, K. D.; Kolk, A. \& Kanatas, A. Outcome in adult patients with head and neck sarcomas--a 10-year analysis. J. Surg. Oncol., 102(2):170-4, 2010.

Müller-Richter, U. D. A.; Katinka Kohlhof, J.; Reichert, T. E. \& Roldán, J. C. Undifferentiated pleomorphic sarcoma of the orbital region. Br. J. Oral Maxillofac. Surg., 46(4):3257, 2008.

Park, S. W.; Kim, H. J.; Lee, J. H. \& Ko, Y. H. Malignant fibrous histiocytoma of the head and neck: CT and MR imaging findings. AJNR Am. J. Neuroradiol., 30(1):71-6, 2009 .

Sarrami, A. H.; Setareh, M.; Afshar-Moghaddam, N.; Izadinejad, M. \& Saadatnia, M. A case of intracranial malignant fibrous histiocytoma. J. Res. Med. Sci., 16(7):968-73, 2011
Shellenberger, T. D. \& Sturgis, E. M. Sarcomas of the head and neck region. Curr. Oncol. Rep., 11(2):135-42, 2009.

Tanaka, T.; Kobayashi, T. \& lino, M. Transformation of benign fibrous histiocytoma into malignant fibrous histiocytoma in the mandible: case report. J. Oral Maxillofac. Surg., 69(7):e285-90, 2011.

Tejani, M. A.; Galloway, T. J.; Lango, M.; Ridge, J. A. \& von Mehren, M. Head and neck sarcomas: a comprehensive cancer center experience. Cancers (Basel), 5(3):890-900, 2013.

Vuity, D.; Bogdan, S.; Csurgay, K.; Sapi, Z.; Nemeth, Z. Malignant fibrous histiocytoma/undifferentiated highgrade pleomorphic sarcoma of the maxillary sinus: report of a case and review of the literature. Pathol. Oncol. Res., 19(4):605-9, 2013

Dirección para Correspondencia:

Dr. Daniel Betancur Castro

Cirujano Dentista

Facultad de Odontología

Universidad de Concepción

Concepción

CHILE

Email: danielbeta.c@gmail.com

Recibido : 02-09-2014

Aceptado: 30-01-2015 